\title{
THE TWIN-NUCLEUS MERGING GALAXY MKN 266
}

\author{
J.B.Hutchings, Dominion Astrophysical Observatory, Victoria, B.C. \\ S.G.Neff, Goddard Space Flight Center, Greenbelt, MD \\ J.H.van Gorkom,Dept of Astronomy,Columbia University, NY
}

\section{Data}

We present results of observations of the double-nucleus galaxy Markarian 266 (NGC 5256) from 3 principal sources. These are

1: CCD imaging with the Canada-France-Hawaii telescope in broad and narrow bands. These indicate that the galaxy has extended, complex, faint outer plumes which indicate that a recent merger has occurred. The narrow-band images reveal remarkable knotty structure of the [O III] emitting gas, extending over the whole central part of the galaxy. This is not seen in $\mathrm{H} \alpha$ (see figure 1).

2: $21 \mathrm{~cm}$ imaging with the VLA, covering velocity space near that of the optical nuclei. The continuum image reveals resolved triple structure, with the two outer peaks coincident with the optical nuclei. The $21 \mathrm{~cm}$ velocity profiles indicate the presence of considerable $\mathrm{H}$ I absorption near the optical emission line velocities.

3: Spatially resolved optical spectroscopy with the DAO $1.8 \mathrm{~m}$ telescope. These data reveal the details of the [O III] velocity field and some of the physical parameters of the gas. The complexity and extended nature of the gas explains some conflicting redshift measurements in the literature. Together with the imaging data, we derive estimates of nuclear reddening and luminosity.

\section{Discussion.}

The deep optical imaging has shown the presence of outer plumes and structure indicative of recent tidal interactions. The presence of two optical nuclei, each with a spiral trail of blue stars and ionised gas suggests that these are the nuclei of two galaxies which are spiralling together. The nuclear activity is not extreme in either case, although nucleus 2 appears to be somewhat reddened. Since nucleus 2 has a Seyfert 2 spectrum, and is probably intrinsically more luminous, it is possible that the [O III] gas is ionised by this nucleus along optical paths which are not obscured. It has been suggested that some (maybe all) Sy 2 nuclei appear to be Sy 1 nuclei whose broad-line (inner) regions are obscured by a ring of material. In this model, the unobscured nuclear radiation is emitted along the polar directions of the ring. The observed $\mathrm{H}$ I must originate in a region shielded from the nuclear radiation. It is clear from the overlapping velocity systems seen in the emission line gas that the detailed velocity field is complex. The observed structure must result from variations in density as well as variable obscuration and/or beaming of the ionising flux. 
Two of the radio components appear to be slightly resolved, indicating that some of the radio emission may be associated with the stellar population rather than arising only from the central object(s). The central radio component is the most flattened and has no optical counterpart; the others lie on the optical nuclei. We note that NGC4038/9 ("the antennae') contain several knots of radio emission, and no known AGN: however, in this system the compact radio emission appears to be thermal and largely associated with $\mathrm{H} \alpha$ emission. The central radio component in Mkn 266 is not associated with $\mathrm{H} \alpha$ emission (see Figure 1).

It has been suggested that the IR ultraluminous galaxies are the earliest stage in the activation of QSOs and AGN. In this stage, the nucleus is enshrouded with dust, and the luminosity is seen principally at IR wavelengths. We have elsewhere suggested that a subgroup of similarly luminous IR sources ('IR QSOs') with flatter spectral indices ( - 0.8$)$ represent a slightly later stage of the process between these and normal (non IR-discovered) QSOs.

The IR luminosity of Mkn 266 is an order of magnitude lower than the ultraluminous IR galaxies, and the optical luminosity is weaker than normal QSOs even after correcting for reddening. However, the IR spectral index is steep, as in the ultraluminous IR galaxies. We have compared the continuum spectrum from radio to optical wavelengths with the spectrum of the IR ultra-luminous galaxy Arp 220. Although Mkn 266 is less luminous than Arp 220 at all frequencies, the basic spectral shapes are remarkably similar. We note also the similarity with Arp 220 in the presence of broad and infalling $\mathrm{H} \mathrm{I}$ absorption and in the presence of strong and clumpy nuclear obscuration. We note that a large HI mass has been inferred in Mkn 266 from CO observations (in fact comparable with that of Arp 220). All this suggests that the basic processes occurring in both systems are the same, and that Mkn 266 is merely a lower-luminosity version of the class of IR-ultraluminous objects. We therefore suggest that Mkn 266 is in the same short-lived initial IR-luminous stage, and expect that it will evolve into a lower luminosity version of the AGN phenomenon.

These results are published in full in the Astronomical Journal.

\section{MKN 266}

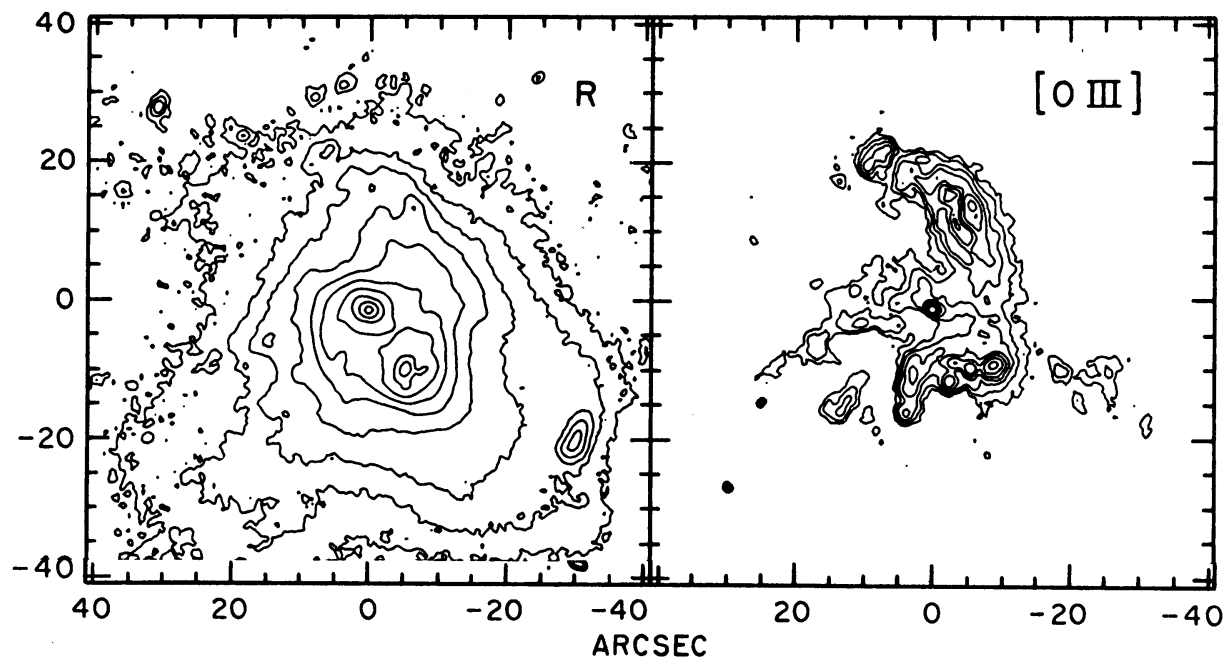

Figure 1. $R$ band and narrow band [O III] images of Mkn 266. 